\title{
A Data-Driven Control Design Approach for Freeway Traffic Ramp Metering with Virtual Reference Feedback Tuning
}

\author{
Shangtai Jin, ${ }^{1}$ Zhongsheng Hou, $^{1}$ Ronghu Chi, ${ }^{2}$ and Jiangen Hao ${ }^{1}$ \\ ${ }^{1}$ Advanced Control Systems Lab, Beijing Jiaotong University, Beijing 100044, China \\ ${ }^{2}$ College of Automation and Electronic Engineering, Qingdao University of Science and Technology, \\ Qingdao 266042, China
}

Correspondence should be addressed to Shangtai Jin; shtjin@bjtu.edu.cn

Received 7 November 2013; Revised 30 December 2013; Accepted 13 January 2014; Published 19 February 2014

Academic Editor: Xiaojie Su

Copyright (C) 2014 Shangtai Jin et al. This is an open access article distributed under the Creative Commons Attribution License, which permits unrestricted use, distribution, and reproduction in any medium, provided the original work is properly cited.

\begin{abstract}
ALINEA is a simple, efficient, and easily implemented ramp metering strategy. Virtual reference feedback tuning (VRFT) is most suitable for many practical systems since it is a "one-shot" data-driven control design methodology. This paper presents an application of VRFT to a ramp metering problem of freeway traffic system. When there is not enough prior knowledge of the controlled system to select a proper parameter of ALINEA, the VRFT approach is used to optimize the ALINEA's parameter by only using a batch of input and output data collected from the freeway traffic system. The extensive simulations are built on both the macroscopic MATLAB platform and the microscopic PARAMICS platform to show the effectiveness and applicability of the proposed data-driven controller tuning approach.
\end{abstract}

\section{Introduction}

Freeway traffic control has become an important area in the field of traffic engineering and intelligent transportation systems due to the rapid expansion in worldwide development of freeway infrastructure and traffic demand. The frequent occurrence of congestions on freeway during rush hours, which may be caused by traffic demand being greater than capacity, traffic accidents, road works or weather, and so forth, leads to travel time delay, inefficient utilization of the freeway infrastructure, and decreasing traffic safety. Thus, freeway traffic control methods should be developed to prevent traffic jam and utilize the freeway infrastructure efficiently. In general, there are three typical freeway traffic control methods, ramp metering, mainline speed control, and corridor control.

Among these methods, ramp metering is the most popular one $[1,2]$. Ramp metering is implemented by means of traffic lights, which is used to meter the number of entering vehicles and prevent traffic volume from exceeding freeway capacity. Ramp metering, when properly applied, is an effective way to ease freeway congestion and improve the efficiency of freeway utilization. From the viewpoint of system control, it is a typical set-point problem and numerous model based control methods have been exploited, such as numerical methods $[1,3]$, linearization method [4], one-step ahead prediction, and multiple prediction adaptive control [5]. However, as the freeway traffic system is expanded to be larger and larger, its accurate mathematical model may be difficult to be built. Therefore, it is desirable to develop a control method that is less dependent on the model accuracy.

In the field of control theory, several control methods for systems with exogenous disturbances and/or model uncertainties have been explored extensively. In [6], a new model transformation of discrete-time fuzzy systems with time-varying delays is analyzed and applied to dynamic output feedback controller design. In [7], a dissipativity based sliding mode control (SMC) is proposed for continuoustime switched stochastic systems with an external disturbance/ uncertainty. In [8], a sliding mode control (SMC) is proposed for Markovian jump singular time-delay systems. In [9], a stable robust model predictive controller with hard input constraints is designed for a multivariable system whose model is inaccurate. In [10], a fuzzy sliding model control method is presented for a class of 
nonlinear systems with structured and unstructured uncertainties. Although the robust performance to disturbances can be achieved with these control methods, the nominal model or system structure is still required for the controller design.

More recently, several data-driven control methods, which focus on designing controller merely using measured input and output data of a plant, are found in the datadriven control field, such as PID control [11], model-free adaptive control [12-14], iterative learning control [15-19], unfalsified control [20], virtual reference feedback tuning [21-24], and iterative feedback tuning [25-27]. Note that ALINEA-a simple, efficient, and easily implemented ramp metering strategy-is a typical PI-type feedback regulator based on mainstream measurements of occupancy downstream of the ramp $[28,29]$. Apparently, it is also a data-driven control strategy in nature without including any modeling information of the freeway system, but only depending on the input and output measurements. However, it is worth pointing out that successful implementation of ALINEA depends on four parameters [30]: the update cycle and the feedback gain of ALINEA controller, the feedback gain, the location, and the predefined optimal occupancy of freeway downstream of the merge. In fact, it is difficult to select an optimal feedback gain by trial-and-error method for a practical freeway traffic system if there is not enough prior knowledge of the controlled system.

In [30], an iterative feedback tuning (IFT) method is proposed to optimize the parameter of the ALINEA controller. The parameter of ALINEA controller is tuned iteratively only by using the input and output (I/O) data without any information of the plant model. However, IFT requires many experiments on the plant for data collection and suffers from local minima problems if it is not suitably initialized [24]. In contrast, VRFT [21-24] is a "one-shot" data-driven controller tuning method: one collects a batch of data from the plant and the procedure returns a controller, without requiring iterations and/or further accesses to the plant for experiments. VRFT formulates the controller tuning problem as a controller parameter identification problem by introducing virtual reference signal. VRFT is suitable for many practical applications since the minimization is conducted in one shot.

In this paper, VRFT method is applied to optimize the parameter of the ALINEA controller in the presence of the modeling uncertainties and exogenous disturbances, as an alternative to the difficult task of fine-tuning ALINEA in realworld testing. The ALINEA's parameter is tuned directly by using the measured input and output $(\mathrm{I} / \mathrm{O})$ data without any prior knowledge of the freeway traffic system. The effectiveness of the proposed data-driven controller tuning method is verified by simulations built on macroscopic MALAB and microscopic PARAMICS platforms.

The paper is organized as follows. Section 2 is the problem formulation, where a discretized macroscopic traffic mode is introduced. Section 3 describes VRFT approach for ALINEA. Simulation results with MATLAB and PARAMICS platforms are provided in Section 4. Finally, Section 5 concludes this paper.

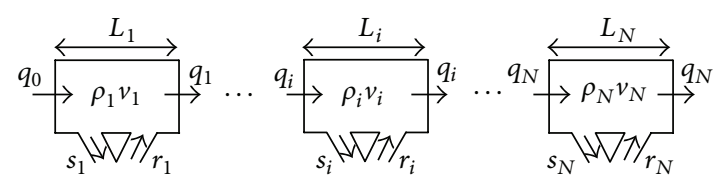

Figure 1: A freeway segment subdivided into sections.

\section{Problem Formulation}

2.1. Macroscopic Traffic Model. The space and time discretized traffic flow model used in this section was proposed by Papageorgiou in 1989. It divides a freeway into several segments, and each segment contains one on-ramp and one off-ramp only, as shown in Figure 1.

The mathematical formulation of discretized traffic flow model is given as follows:

$$
\begin{aligned}
& \rho_{i}(k+1)=\rho_{i}(k)+\frac{T}{L_{i}}\left[q_{i-1}(k)-q_{i}(k)+r_{i}(k)-s_{i}(k)\right], \\
& q_{i}(k)=\rho_{i}(k) v_{i}(k), \\
& v_{i}(k+1)=v_{i}(k)+\frac{T}{\tau}\left[V\left(\rho_{i}(k)\right)-v_{i}(k)\right] \\
& +\frac{T}{L_{i}} v_{i}(k)\left[v_{i-1}(k)-v_{i}(k)\right] \\
& -\frac{\gamma T}{\tau L_{i}} \frac{\left[\rho_{i+1}(k)-\rho_{i}(k)\right]}{\left[\rho_{i}(k)+\kappa\right]}, \\
& V\left(\rho_{i}(k)\right)=v_{\text {free }}\left(1-\left[\frac{\rho_{i}(k)}{\rho_{\text {jam }}}\right]^{l}\right)^{m},
\end{aligned}
$$

where $T$ is the sample time interval in hour. $k$ is the $k$ th time interval, $i \in\{1, \ldots, N\}$ is the $i$ th section of a freeway, and $N$ is the total section number. Model variables are listed as follows:

$\rho_{i}(k)$ : density in section $i$ at time $k T($ veh $/$ lane $/ \mathrm{km})$;

$v_{i}(k)$ : space mean speed in section $i$ at time $k T(\mathrm{~km} / \mathrm{h})$;

$q_{i}(k)$ : traffic flow leaving section $i$ and entering section $i+1$ at time $k T(\mathrm{veh} / \mathrm{h})$;

$r_{i}(k)$ : on-ramp traffic volume for section $i$ at time $k T$ (veh/h);

$s_{i}(k)$ : off-ramp traffic volume for section $i$ at time $k T(\mathrm{veh} / \mathrm{h})$, which is regarded as an unknown disturbance;

$L_{i}$ : length of freeway in section $i(\mathrm{~km})$;

$v_{\text {free }}(\mathrm{km} / \mathrm{h})$ and $\rho_{\text {jam }}(\mathrm{veh} / \mathrm{lane} / \mathrm{km})$ : the free speed and the maximum possible density per lane, respectively. They are two important parameters in traffic flow model, since their accuracy affects the accuracy of traffic flow model;

$\tau, \gamma, \kappa, l$, and $m$ : constant parameters which reflect particular characteristics of a given traffic system and 
depend upon the freeway geometry, vehicle characteristics, drivers' behaviors, and so forth.

Equations (1)-(4) constitute the macroscopic traffic model. Equation (1) is the well-known conservation equation, (2) is the flow equation, (3) is the empirical dynamic speed equation, and (4) represents the density-dependent equilibrium speed.

2.2. Boundary Conditions. We assume that the traffic flow rate entering section 1 during the time period $k T$ and $(k+1) T$ is $q_{0}(k)$ and the mean speed of the traffic entering section 1 is equal to the mean speed of section 1 ; that is, $v_{0}(k)=v_{1}(k)$. We also assume that the mean speed and traffic density of the traffic exiting section $N+1$ are equal to those of section $N$; that is, $v_{N+1}(k)=v_{N}(k), \rho_{N+1}(k)=\rho_{N}(k)$. Boundary conditions can be summarized as follows:

$$
\begin{aligned}
\rho_{0}(k) & =\frac{q_{0}(k)}{v_{1}(k)}, \\
v_{0}(k) & =v_{1}(k), \\
\rho_{N+1}(k) & =\rho_{N}(k), \\
v_{N+1}(k) & =v_{N}(k), \quad \forall k .
\end{aligned}
$$

2.3. Control Objective. For the traffic system, the control objective is to seek an appropriate on-ramp traffic volume $r_{i}(k)$ such that the traffic density $\rho_{i}(k)$ tracks the desired traffic density $\rho_{d}$. It is worth to point out that the offramp traffic volume $s_{i}(k)$ is an uncontrollable variable and is regarded as exogenous disturbance here. Obviously, even though the freeway model is known, it is difficult to design a proper control law using the traditional model-based control approaches such as optimal control and adaptive control because of the strong nonlinearity and uncertainties in the freeway traffic flow model.

For the simplicity of formulation, the section index $i$ is omitted in the following equations.

\section{Virtual Reference Feedback Tuning for ALINEA Controller}

3.1. ALINEA Controller. Reactive ramp metering strategies are employed at a tactical level, that is, in the aim of keeping the freeway traffic conditions close to prespecified set values, based on real-time measurements. The occupancy strategy is based on the same philosophy as the demand-capacity strategy, but it relies on occupancy-based estimation of the freeway flow measurement upstream of the ramp, which may, under certain conditions, reduce the corresponding implementation cost. Since the concept of occupancy and traffic density is similar and has a linear proportion between them, in this paper, we use the traffic density instead of the occupancy to design the ALINEA controller as follows [30]:

$$
r(k)=r(k-1)+\Theta\left[\rho_{d}-\rho(k)\right],
$$

where $\rho_{d}$ is the desired traffic density and $\Theta$ is the feedback gain of ALINEA controller.

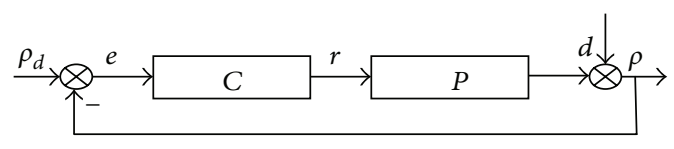

FIGURE 2: The closed-loop freeway traffic system.

3.2. VRFT Approach. Consider the freeway traffic system. As shown in Figure 2, it is a classical one-degree-of-freedom control system, where $P$ is the freeway traffic system and $\mathrm{C}$ is the ALINEA controller. $\rho_{d}, \rho, r, d$, and $e$ are the reference traffic density, the traffic density, the ramp metering volume, disturbance, and the difference between $\rho_{d}$ and $\rho$, respectively. The closed-loop freeway traffic system is described as

$$
\begin{gathered}
\rho(k)=P(z) r(k), \\
r(k)=\mathrm{C}(z, \Theta)\left(\rho_{d}-\rho(k)\right),
\end{gathered}
$$

where $z$ is the one-step ahead shift operator and $\Theta$ is the controller parameter.

The transfer function of the closed-loop system can be rewritten as

$$
\frac{P(z) \mathrm{C}(z, \Theta)}{1+P(z) \mathrm{C}(z, \Theta)} .
$$

For an unknown freeway traffic system $P(z)$, the control objective is to find an optimal controller parameter $\Theta_{\text {opt }}$ by using a batch of the measured input/output data so that the freeway traffic system behavior approximates as much as possible to that of a given invertible reference model $M(z)$, where $z$ is the one-step ahead shift operator [21-24]. This can be achieved by minimizing the following model-reference criterion:

$$
J(\Theta)=\left\|\left(\frac{P(z) \mathrm{C}(z, \Theta)}{1+P(z) \mathrm{C}(z, \Theta)}-M(z)\right) W(z)\right\|_{2}^{2},
$$

where $W(z)$ is a weighting function.

It is difficult to calculate the derivative of the criterion (9) with respect to controller parameter $\Theta$ if the freeway traffic system $P(z)$ is unknown. To address this issue, one can introduce a virtual reference density signal $\rho_{\text {vir }}(k)$ such that

$$
\rho(k)=M(z) \rho_{\mathrm{vir}}(k)
$$

where $\rho_{\text {vir }}(k)$ does not exist in reality and was not used in the generation of $\rho(k)$.

Since $M(z)$ is a given invertible reference model, (10) is rewritten as

$$
\rho_{\text {vir }}(k)=M(z)^{-1} \rho(k),
$$

where $M(z)^{-1}$ is the inversion of $M(z)$.

Equation (10) implies that $\rho(k)$ is the desired density of the freeway system if the reference density signal is set as $\rho_{\text {vir }}(k)$ and the corresponding virtual tracking error is $e_{\mathrm{vir}}(k)=\rho_{\mathrm{vir}}(k)-\rho(k)$. On the other hand, even though the freeway system is unknown, when the freeway traffic system 
TABLE 1: Initial values and parameters associated with the traffic model [18, 19].

\begin{tabular}{|c|c|c|c|c|c|c|c|c|c|c|c|c|}
\hline Section & 1 & 2 & 3 & 4 & 5 & 6 & 7 & 8 & 9 & 10 & 11 & 12 \\
\hline$\rho_{i}(0)$ & 30 & 30 & 30 & 30 & 30 & 30 & 30 & 30 & 30 & 30 & 30 & 30 \\
\hline$v_{i}(0)$ & 50 & 50 & 50 & 50 & 50 & 50 & 50 & 50 & 50 & 50 & 50 & 50 \\
\hline \multirow{2}{*}{ Parameters } & $v_{\text {free }}$ & $\rho_{\mathrm{jam}}$ & $l$ & $m$ & $\kappa$ & $\tau$ & $T$ & $\gamma$ & $q_{0}(k)$ & $r_{i}(0)$ & $\alpha$ & \\
\hline & $80 \mathrm{~km} / \mathrm{h}$ & $80 \mathrm{veh} / \mathrm{lane} / \mathrm{km}$ & 1.8 & 1.7 & $13 \mathrm{veh} / \mathrm{km}$ & $0.01 \mathrm{~h}$ & $0.00417 \mathrm{~h}$ & $35 \mathrm{~km}^{2} / \mathrm{h}$ & $1500 \mathrm{veh} / \mathrm{h}$ & $0 \mathrm{veh} / \mathrm{h}$ & 0.95 & \\
\hline
\end{tabular}

is fed by $r(k)$ (the actually measured ramp metering volume), it generates $\rho(k)$ (the corresponding measured output signal). Thus, if the reference signal is set to be the virtual reference density signal $\rho_{\text {vir }}(k)$ and the corresponding virtual tracking error is $e_{\mathrm{vir}}(k)$, a good controller must generate the ramp metering signal $r(k)$. Since both the signals $e_{\mathrm{vir}}(k)$ and $r(k)$ are available, the control objective (9) can be transformed into the following standard identification problem:

$$
J_{\mathrm{VRFT}}(\Theta)=\left\|L(z)\left(\mathrm{C}(z, \Theta) e_{\mathrm{vir}}-r\right)\right\|^{2},
$$

where $L(z)$ is a suitable filter.

Remark 1. For a practical control problem, the "ideal controller" is usually a complex nonlinear system and it does not belong to the given controller class. In [24], the filter $L(z)$ is introduced to deal with this problem. Minimizing $J_{\mathrm{VRFT}}(\Theta)$ with the filter will generate a "nearly minimizer" of $J(\Theta)$. It is proved that minimizing $J_{\mathrm{VRFT}}(\Theta)$ is equivalent to minimizing the second-order expansion of $J(\Theta)$ in a constrained sense.

The procedure of VRFT for ALINEA controller is summarized as follows:

(1) collect a batch of input/output data collected from the plant, expressed as $\left\{(r(k), \rho(k))_{k=1: N}\right\}$, where $N$ denotes the number of the input/output data pairs;

(2) calculate the virtual reference density signal $\left\{\left(\rho_{\text {vir }}(k)\right)_{k=1: N}\right\}$ for a given invertible $M(z)$ and the measured density signal $\left\{\rho(k)_{k=1: N}\right\}$ according to (11);

(3) calculate the virtual error signal $\left\{\left(e_{\text {vir }}(k)\right)_{k=1: N}\right\}$ according to the following equation:

$$
e_{\mathrm{vir}}(k)=\rho_{\mathrm{vir}}(k)-\rho(k) \text {; }
$$

(4) filter the signals $e_{\mathrm{vir}}(k)$ and $r(k)$ with a suitable filter $L(z)$, obtaining $e_{L}(k)=L(z) e_{\mathrm{vir}}(k)$ and $r_{L}(k)=$ $L(z) r(k)$;

(5) estimate the controller parameter

$$
\widehat{\Theta}_{\mathrm{opt}}=\arg \min _{\Theta} J_{\mathrm{VRFT}}^{N}(\Theta),
$$

where

$$
J_{\mathrm{VRFT}}^{N}(\Theta)=\frac{1}{N} \sum_{i=1}^{N}\left(r_{L}(i)-\mathrm{C}(z, \Theta) e_{L}(i)\right)^{2} .
$$

Remark 2. As suggested in [24], the filter can be designed as follows:

$$
L(z)=\left(1-M(z) z^{-1}\right)\left(\left.\frac{\partial P(z)}{\partial u}\right|_{u}\right),
$$

where $\partial P(z) /\left.\partial u\right|_{u}$ is linear and time varying and it can be estimated, for example, via forgetting factor identification techniques.

\section{Illustrative Examples}

MATLAB and PARAMICS are widely used in the area of traffic management and academic research for evaluation and validation. In this section, in order to evaluate the proposed VRFT-tuned ALINEA, two simulations are carried out on MATLAB and PARAMICS platforms, respectively, where the macroscopic traffic flow model (1)-(4) is simulated on MATLAB platform, and the microscopic traffic flow model is simulated on PARAMICS platform. Both simulations show the effectiveness of the proposed VRFT-tuned ALINEA.

\subsection{Performance Evaluation with MATLAB Platform}

4.1.1. Network Configuration. Consider a long segment of freeway that is divided uniformly into 12 sections. The length of each section is $0.5 \mathrm{~km}$. The initial traffic volume entering section 1 is $1500 \mathrm{veh} / \mathrm{h}$. The desired density is $\rho_{d}(t)=$ $30 \mathrm{veh} /$ lane $/ \mathrm{km}$. The initial density and mean speed of each section are shown in Table 1 and the parameters used in the macroscopic traffic model are also listed in Table 1.

There exist an on-ramp with known traffic demands in section 3 and an off-ramp with unknown exiting traffic flow in section 8 . The traffic demand pattern (on-ramp) and the outflow pattern (off-ramp) are shown in Figure 3. They were chosen to simulate a traffic scenario during rush hour. Note that the queuing demands actually impose a constraint on the control inputs of ramp metering; that is, the on-ramp volumes cannot exceed the current demands plus the existing waiting queues at on-ramp 3 at time $k$; thus

$$
r_{3}(k) \leq d_{3}(k)+l_{3}(k),
$$

where $l_{3}(k)$ denotes the length (in vehicles) of a possibly existing waiting queue at time $k$ at $3 \mathrm{rd}$ on-ramp and $d_{3}(t)$ is the demand flow at time $k$ at 3rd on-ramp (veh/h).

On the other hand, the waiting queue is the accumulation of the difference between the demand and actual on-ramp; that is,

$$
l_{3}(k+1)=l_{3}(k)+T\left(d_{3}(k)-r_{3}(k)\right) .
$$




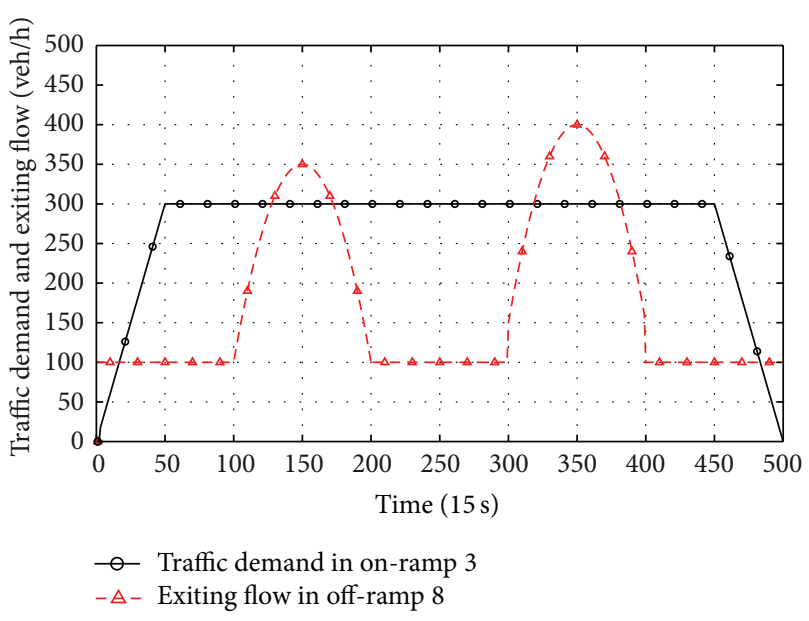

Figure 3: Traffic demand in on-ramp 3 and exiting flow of off-ramp 8.

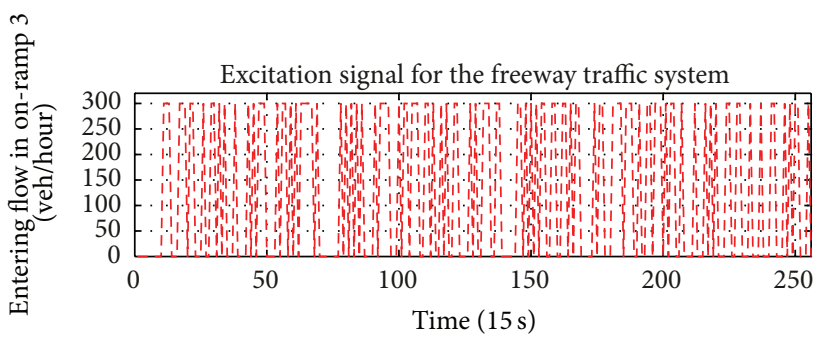

(a)

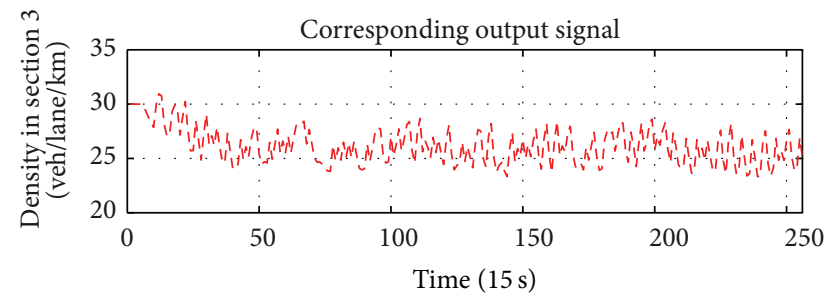

(b)

FIGURE 4: The open-loop I/O signals measured on the system.

4.1.2. Simulation and Results. Using the VRFT method presented in Section 3, the ALINEA controller can be straightforwardly designed using the I/O data measured on the freeway traffic system. Specifically, the control input signal used for open-loop excitation is a pseudorandom binary sequence signal sampled at 15 seconds. The length of the data vector is 256 (corresponding to 1.068 hour of data acquisition). The I/O signals measured on the system are displayed in Figure 4. According to the characteristics of the measured input signal, the filter for VRFT method is simply set to be $L(z)=1$.

The VRFT toolbox for MATLAB 6 Release 13 [31] is used to tune the controller parameter $\Theta$. For a given first-order reference model $M(z)=0.9 z^{-1} /\left(1-0.1 z^{-1}\right)$, a filter $L(z)=$ 1 , a weighting function $W(z)=1$, and a batch of measured input and output data, VRFT toolbox gives the optimal feedback gain $\Theta_{\text {opt }}=50.75$.

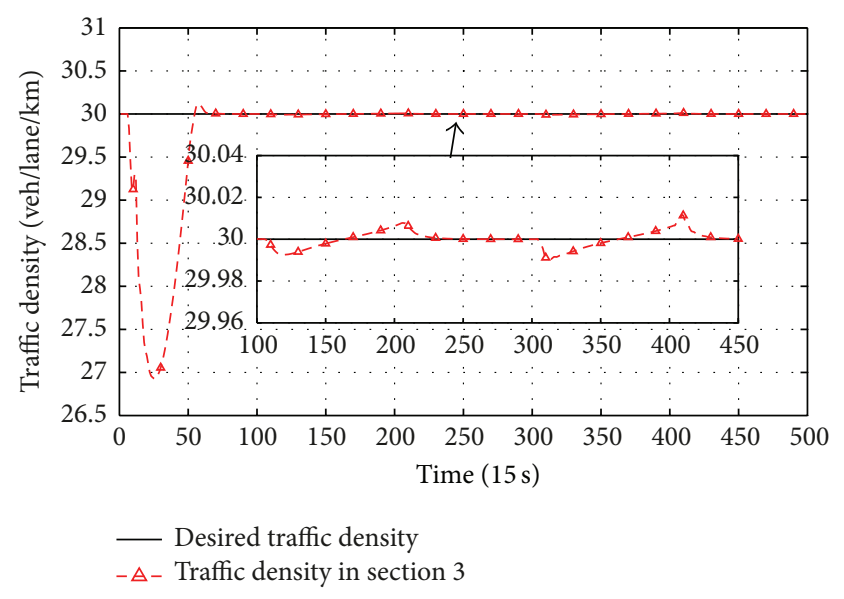

(a) Traffic density in section 3

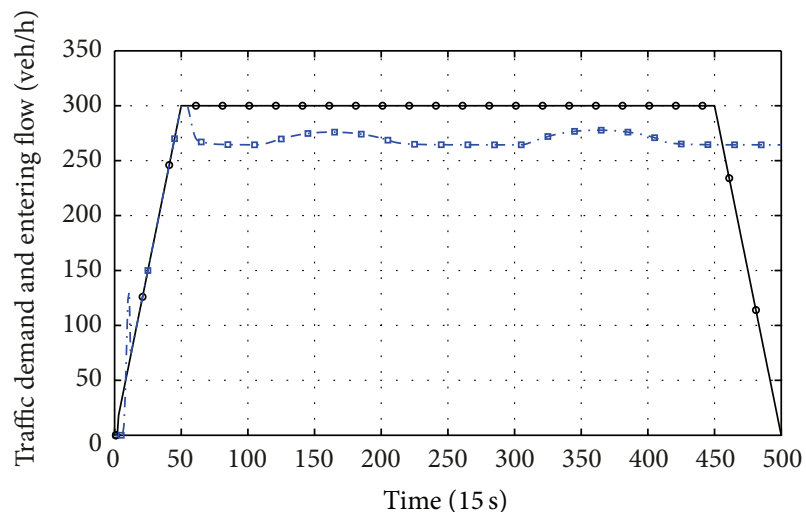

- - Traffic demand in on-ramp 3

$-\square-$ Entering flow in on-ramp 3

(b) Traffic demand and entering flow in on-ramp 3

Figure 5: Control performance of VRFT-tuned ALINEA.

The control performance of ALINEA controller with feedback gain $\Theta_{\text {opt }}=50.75$ is quite well despite the unknown disturbance in off-ramp 8, as shown in Figure 5, where Figure 5(a) shows the density profile in section 3 and Figure 5(b) shows the entering flow in on-ramp 3. The simulation results illustrate that a proper ALINEA's parameter is obtained by using the VRFT method presented in this paper.

4.2. Performance Evaluation in PARAMICS Platform. Existing microscopic traffic simulation platforms have distinguished features, and the fundamental model is commonly the car following model, which makes simulations very similar to each other. In this paper, we adopt PARAMICS simulation platform, which is widely used in the area of traffic management and academic research for evaluation and validation.

4.2.1. Freeway Network. A single lane freeway link with 14 mainline sections, 1 on-ramp, and 1 off-ramp is considered. The on-ramp used to implement metering or flow control is connected to section 3 at the beginning and the off-ramp 


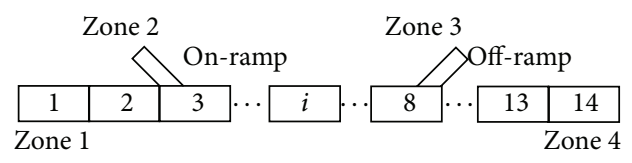

Figure 6: Freeway simulation model.

TABLE 2: PARAMICS O-D table.

\begin{tabular}{lccccc}
\hline & Zone 1 & Zone 2 & Zone 3 & Zone 4 & Total \\
\hline Zone 1 & 0 & 0 & 250 & 1250 & $\mathbf{1 5 0 0}$ \\
Zone 2 & 0 & 0 & 50 & 250 & $\mathbf{3 0 0}$ \\
Zone 3 & 0 & 0 & 0 & 0 & $\mathbf{0}$ \\
Zone 4 & 0 & 0 & 0 & 0 & $\mathbf{0}$ \\
Total & $\mathbf{0}$ & $\mathbf{0}$ & $\mathbf{3 0 0}$ & $\mathbf{1 5 0 0}$ & $\mathbf{1 8 0 0}$ \\
\hline
\end{tabular}

is connected to section 8 at the end. As shown in Figure 6, vehicles enter into the network from two defined zones, Zone 1 and Zone 2, at the beginning of the freeway mainline and onramp section, respectively, and will have their destinations to be either Zone 3 or Zone 4, defined at the end of off-ramp and the mainline.

In Table 2, Zone 1 and Zone 2 are used for origins to release vehicles into the network, and meanwhile Zone 3 and Zone 4 are used as destination for these vehicles. In the table, the number specified is the total number of vehicles expected to make a trip starting from the zone corresponding to the row to the zone corresponding to the column. The release rate in PARAMICS of traffic flow is specified in profile files. The duration time is divided uniformly into time intervals and a specified percentage of vehicles from the total demand are expected to be released from each origin zone during each time interval; additionally the release probability is subject to random process. In this paper the time interval length is set to be 3 minutes which divides the simulation duration of 1 hour into 20 intervals.

4.2.2. Network Configuration. The key parameters for traffic model and simulation are provided in Table 3. In Table 3, duration is the length of the simulation; time step is the number of discrete simulation intervals that are simulated per second; demand factor specifies the dynamic demand for the current simulation ranging from 0 to $200 \%$ of the current global demand; orientation specifies the side of the carriageway that vehicles travel upon (right-/left-hand drive); units specify the unit convention for display in PARAMICS (USA/UK/Metric); control time step specifies the length of time interval for updating of control signal. The rest of parameters are commonly used parameters in the field of control; therefore further explanations are omitted.

4.2.3. Simulation and Results. As for a realistic implementation in the PARAMICS microscopic simulation platform where metered vehicles can only be integer numbers, so a revised ALINEA law of (6) is given as follows:

$$
r(k)=r(k-1)+\operatorname{INT}\left(\Theta\left(\rho_{d}-\rho(k)\right)\right),
$$

where the desired density $\rho_{d}$ is set to be $30 \mathrm{veh} /$ lane $/ \mathrm{km}$.
TABLE 3: Parameters for simulation.

\begin{tabular}{lc}
\hline Duration (HH:MM:SS) & $1: 00: 00$ \\
Time step (second) & 2 \\
Control time step (second) & 30 \\
Demand factor (\%) & 100 \\
Section length (m) & 500 \\
Orientation & Left-hand drive \\
Units & Metric units \\
\hline
\end{tabular}

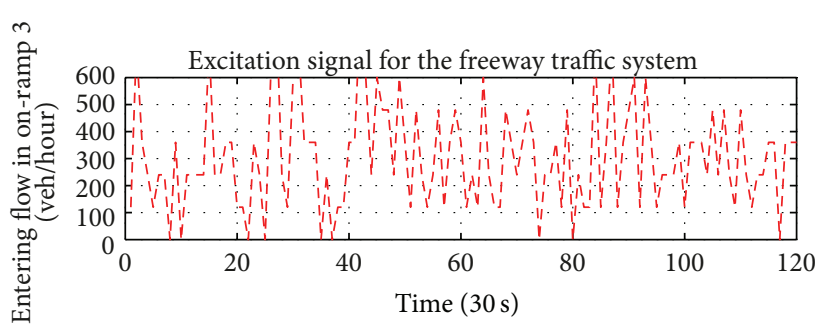

(a)

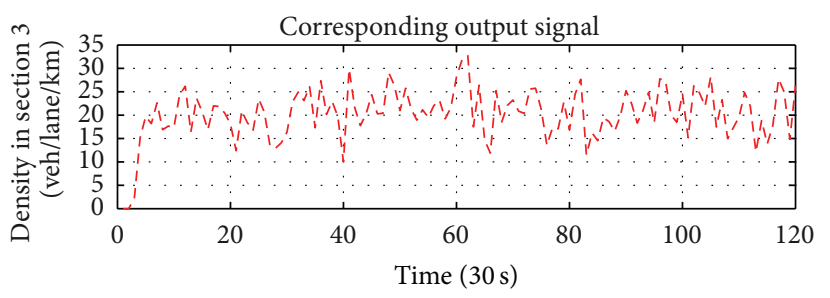

(b)

Figure 7: The open-loop I/O signals measured on the system.

The control input signal used for open-loop excitation is a pseudorandom binary sequence signal sampled at $30 \mathrm{sec}$. The length of the data vector is 100 (corresponding to $50 \mathrm{~min}$ of data acquisition). The I/O signals measured on the system are displayed in Figure 7. According to the characteristics of the measured input signal, the filter for VRFT method is set to be $L(z)=\left(0.9 z^{-1}-1.1006 z^{-2}+0.089625 z^{-3}-0.19983 z^{-4}+\right.$ $\left.0.10872 z^{-5}+0.2021 z^{-6}\right) /\left(1-0.2 z^{-1}+0.01 z^{-2}\right)$. For a given first-order reference model $M(z)=0.9 z^{-1} /\left(1-0.1 z^{-1}\right)$, a weighting function $W(z)=1$, and a batch of measured input and output data, VRFT toolbox gives the optimal feedback gain $\Theta_{\text {opt }}=44.95$. The simulation result is shown in Figure 8 . It is clear that the density tracking performance using the VRFT-tuned ALINEA is satisfactory.

To further evaluate the controller's performance, we define average absolute difference between density and desired density as the performance index:

$$
J=\frac{\sum_{k=T_{1}}^{k=T_{2}}\left|\rho_{d}-\rho(k)\right|}{T_{2}-T_{1}},
$$

where $T_{1}=20$ and $T_{1}=120$ are the beginning and ending time instances that decide the traffic period for evaluation. The final performance index is $J=1.5357$. 


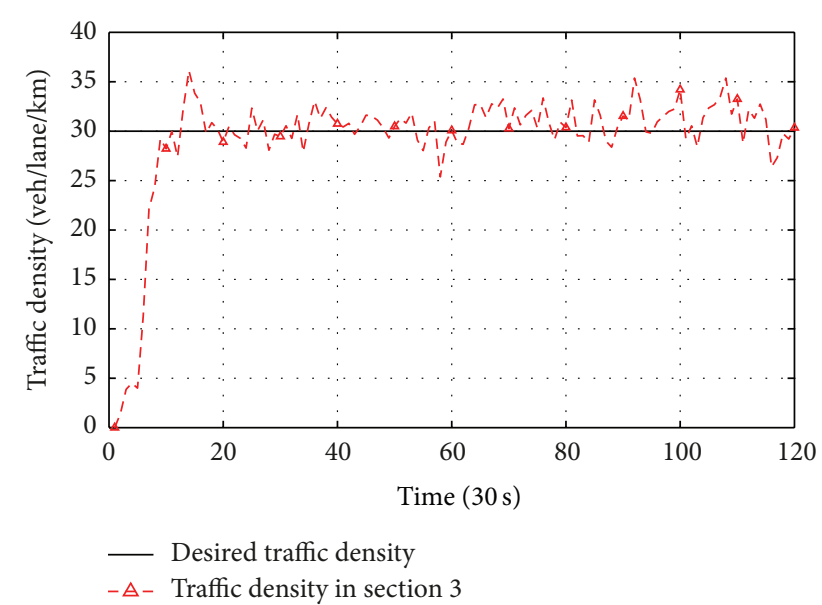

FIgURE 8: Mainline density resulting from VRFT-tuned ALINEA.

\section{Conclusion}

In this paper, a "one-shot" data-driven controller tuning method, VRFT, is applied to tune the parameters in ALINEA controller. It can easily find an optimal feedback gain. The main feature of VRFT is that the method aims at minimizing a cost function by using a batch of input and output data collected from the controlled plant. ALINEA controller tuned by using VRFT method is evaluated on macroscopic MATLAB and microscopic PARAMICS platforms, respectively. The simulation results show the effectiveness of the datadriven tuning approach. It is noted that the parameters, such as the vehicle characteristics, drivers' behaviors, are time varying naturally in the practical traffic network. Therefore, the extension of the VRFT method to time-varying freeway systems will be explored in our future work. Other effective control methods [6-10] to deal with large model uncertainties and exogenous disturbances will also be explored further with applications in freeway traffic systems.

\section{Conflict of Interests}

The authors declare that there is no conflict of interests regarding the publication of this paper.

\section{Acknowledgments}

This work is supported by National Natural Science Foundation of China $(61120106009,61374102)$ and the Fundamental Research Funds for the Central Universities (2014JBM005).

\section{References}

[1] M. Papageorgiou and A. Kotsialos, "Freeway ramp metering: an overview," IEEE Transactions on Intelligent Transportation Systems, vol. 3, no. 4, pp. 271-281, 2002.

[2] P. Varaiya, "Reducing highway congestion: an empirical approach," European Journal of Control, vol. 11, no. 4-5, pp. 301-309, 2005.
[3] A. Kotsialos, M. Papageorgiou, M. Mangeas, and H. Haj-Salem, "Coordinated and integrated control of motorway networks via non-linear optimal control," Transportation Research C, vol. 10, no. 1, pp. 65-84, 2002.

[4] T. H. Chang and Z. Y. Li, "Optimization of mainline traffic via an adaptive co-ordinated ramp-metering control model with dynamic OD estimation," Transportation Research C, vol. 10, no. 2, pp. 99-120, 2002.

[5] H. B. Ji, "Freeway traffic systems: prediction and control," in Proceedings of the IEEE 46th Vehicular Technology Conference on Mobile Technology for the Human Race, vol. 3, pp. 1815-1819, Atlanta, Ga, USA, April-May 1996.

[6] X. J. Su, P. Shi, L. G. Wu, and Y. D. Song, "A novel control design on discrete-time takagi-sugeno fuzzy systems with timevarying delays," IEEE Transactions on Fuzzy Systems, vol. 21, no. 4, pp. 655-671, 2013.

[7] L. G. Wu, W. X. Zheng, and H. J. Gao, "Dissipativity-based sliding mode control of switched stochastic systems," IEEE Transactions on Automatic Control, vol. 58, no. 3, pp. 785-791, 2013.

[8] L. G. Wu, X. J. Su, and P. Shi, "Sliding mode control with bounded $L_{2}$ gain performance of Markovian jump singular timedelay systems," Automatica, vol. 48, no. 8, pp. 1929-1933, 2012.

[9] V. Vesely and J. Osusky, "Robust multivariable generalized predictive control design," International Journal of Innovative Computing, Information and Control, vol. 9, no. 8, pp. 33773390, 2013.

[10] M. R. Soltanpour, B. Zolfaghari, M. Soltani, and M. H. Khooban, "Fuzzy sliding mode control design for a class of nonlinear systems with structured and unstructured uncertainties," International Journal of Innovative Computing, Information and Control, vol. 9, no. 7, pp. 2713-2726, 2013.

[11] G. J. Silva, A. Datta, and S. P. Bhattacharyya, "New results on the synthesis of PID controllers," IEEE Transactions on Automatic Control, vol. 47, no. 2, pp. 241-252, 2002.

[12] Z. S. Hou, Nonparametric Models and Its Adaptive Control Theory, Science Press, Beijing, China, 1999.

[13] Z. S. Hou and S. T. Jin, "A novel data-driven control approach for a class of discrete-time nonlinear systems," IEEE Transactions on Control Systems Technology, vol. 19, no. 6, pp. 1549-1558, 2011.

[14] Z. S. Hou and S. T. Jin, "Data-driven model-free adaptive control for a class of MIMO nonlinear discrete-time systems," IEEE Transactions on Neural Networks, vol. 22, no. 12, pp. 2173-2188, 2011.

[15] S. Arimoto, S. Kawamura, and F. Miyazaki, "Bettering operation of robots by learning," Journal of Robotic Systems, vol. 1, no. 2, pp. 123-140, 1984.

[16] H. S. Ahn, Y. Q. Chen, and K. L. Moore, "Iterative learning control: brief survey and categorization," IEEE Transactions on Systems, Man and Cybernetics C, vol. 37, no. 6, pp. 1099-1121, 2007.

[17] J. X. Xu, "A survey on iterative learning control for nonlinear systems," International Journal of Control, vol. 84, no. 7, pp. 1275-1294, 2011.

[18] Z.S. Hou, J. X. Xu, and J. W. Yan, "An iterative learning approach for density control of freeway traffic flow via ramp metering," Transportation Research C, vol. 16, no. 1, pp. 71-97, 2008.

[19] Z. S. Hou, J. X. Xu, and H. W. Zhong, "Freeway traffic control using iterative learning control-based ramp metering and speed signaling," IEEE Transactions on Vehicular Technology, vol. 56, no. 2, pp. 466-477, 2007. 
[20] M. G. Safonov and T. C. Tsao, "The unfalsified control concept: a direct path from experiment to controller," in Feedback Control, Nonlinear Systems, and Complexity, vol. 202 of Lecture Notes in Control and Information Sciences, pp. 196-214, Springer, London, UK, 1995.

[21] S. M. Savaresi and G. O. Guardabassi, "Approximate I/O feedback linearization of discrete-time non-linear systems via virtual input direct design," Automatica, vol. 34, no. 6, pp. 715-722, 1998.

[22] M. C. Campi, A. Lecchini, and S. M. Savaresi, "Virtual reference feedback tuning (VRFT): a new direct approach to the design of feedback controllers," in Proceedings of the 39th IEEE Confernce on Decision and Control, pp. 623-629, Sydney, Australia, December 2000.

[23] M. C. Campi, A. Lecchini, and S. M. Savaresi, "Virtual reference feedback tuning: a direct method for the design of feedback controllers," Automatica, vol. 38, no. 8, pp. 1337-1346, 2002.

[24] M. C. Campi and S. M. Savaresi, "Direct nonlinear control desaign: the virtual reference feedback tuning (VRFT) approach," IEEE Transactions on Automatic Control, vol. 51, no. 1, pp. 14-27, 2006.

[25] H. Hjalmarsson, M. Gevers, S. Gunnarsson, and O. Lequin, "Iterative feedback tuning: theory and applications," IEEE Control Systems Magazine, vol. 18, no. 4, pp. 26-41, 1998.

[26] H. Hjalmarsson, "Iterative feedback tuning-an overview," International Journal of Adaptive Control and Signal Processing, vol. 16, no. 5, pp. 373-395, 2002.

[27] H. Hjalmarsson, "From experiment design to closed-loop control," Automatica, vol. 41, no. 3, pp. 393-438, 2005.

[28] M. Papageorgiou, H. Hadj-Salem, and J. M. Blosseville, "ALINEA: a local feedback control law for on-ramp metering," Transportation Research Record, no. 1320, pp. 58-64, 1991.

[29] M. Papageorgiou, H. Hadj-Salem, and F. Middelham, "ALINEA local ramp metering: summary of field results," Transportation Research Record, no. 1603, pp. 90-98, 1997.

[30] R. H. Chi, Z. S. Hou, S. T. Jin, and D. W. Wang, "A data-driven iterative feedback tuning approach of ALINEA for freeway traffic ramp metering with PARAMICS simulations," IEEE Transactions on Industrial Informatics, vol. 9, no. 4, pp. 23102317, 2013.

[31] http://www.ing.unibs.it/campi/VRFTwebsite/index.html. 


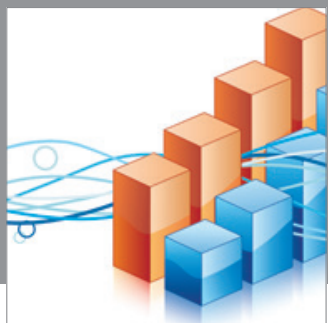

Advances in

Operations Research

mansans

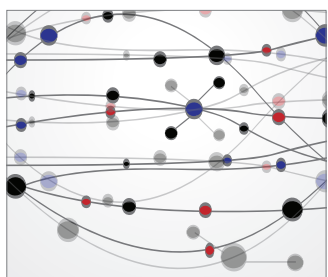

The Scientific World Journal
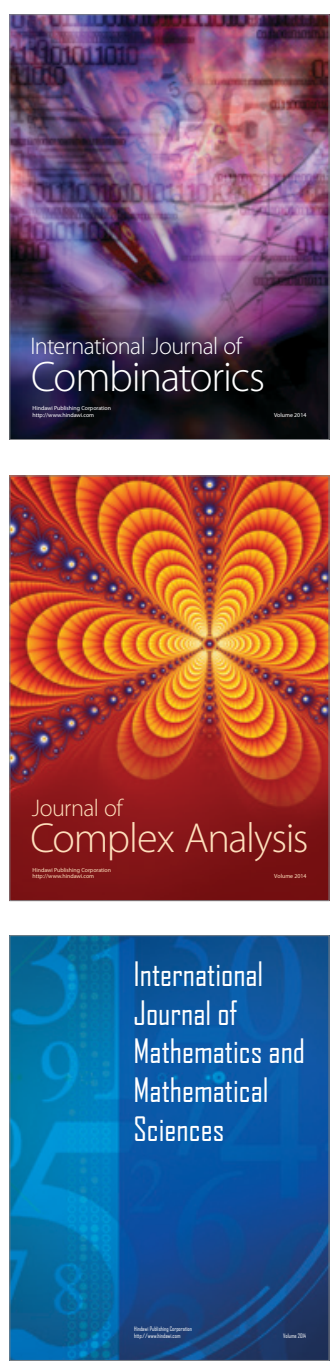
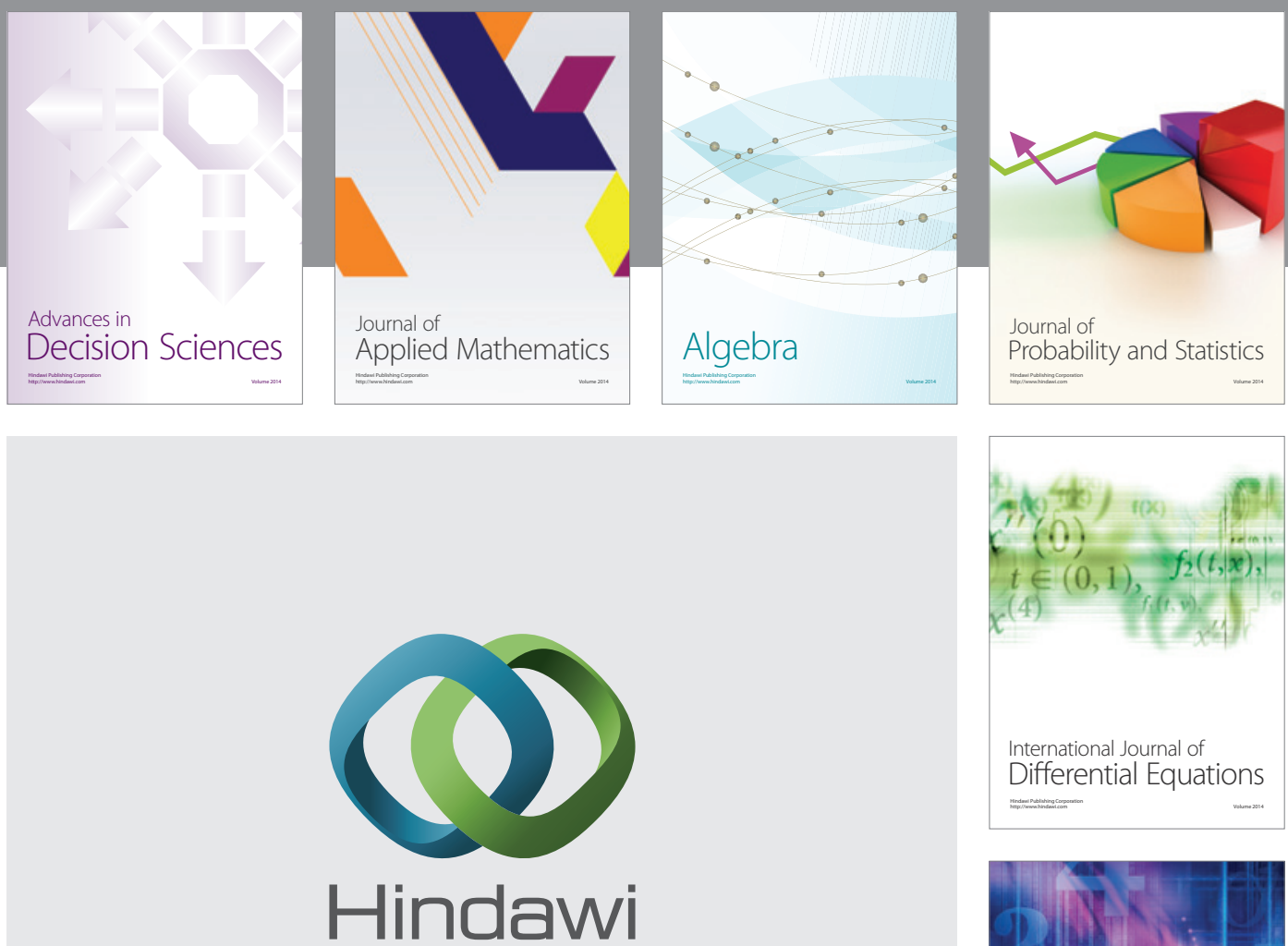

Submit your manuscripts at http://www.hindawi.com
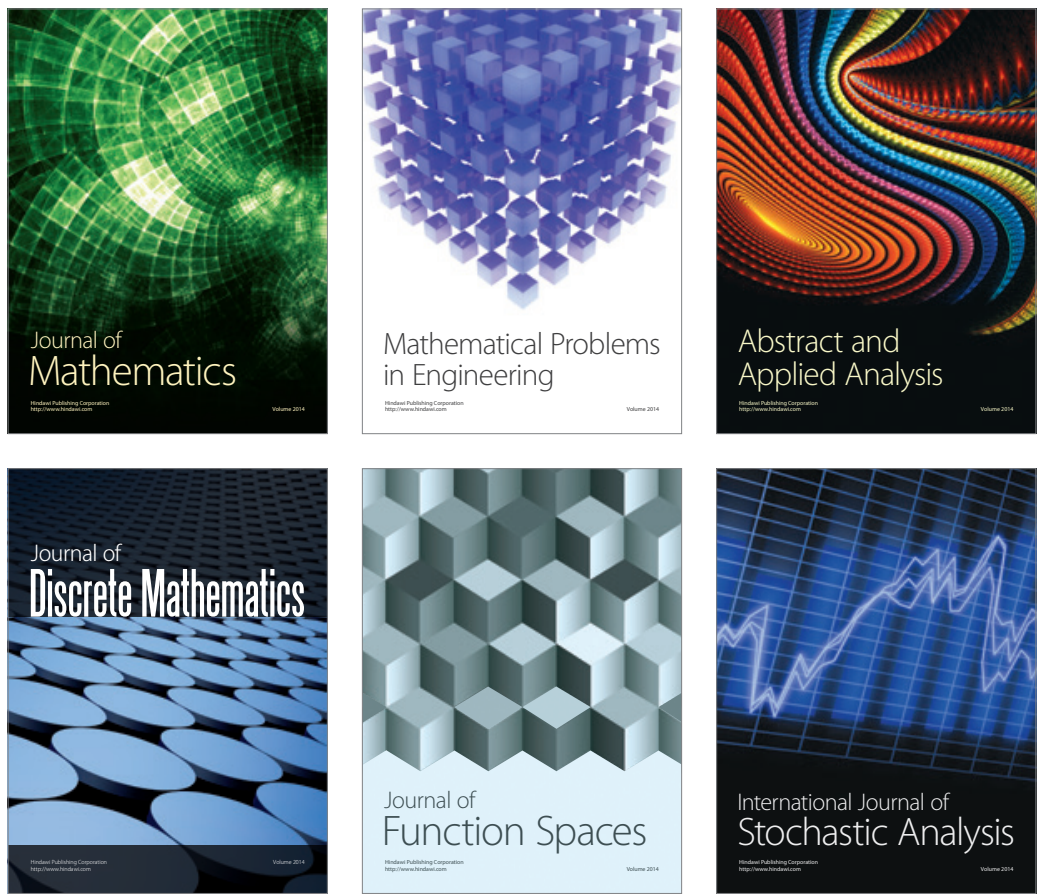

Journal of

Function Spaces

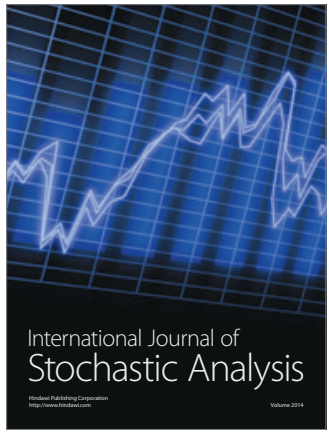

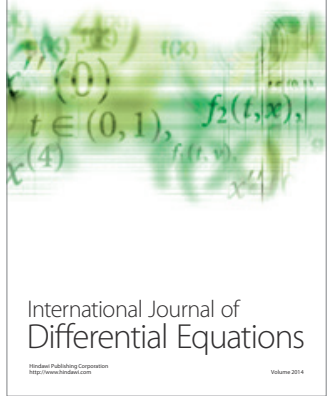
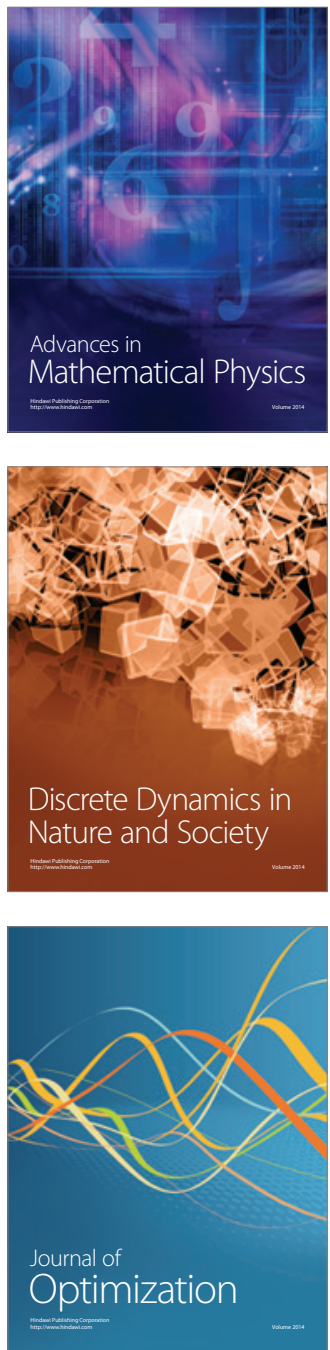